\title{
Protocol proposal for the invasive approach of the popliteal muscle
}

\author{
Bosch Janer R. ${ }^{1}$ Togores Morlà B. ${ }^{2}$ Cortés Servera J. ${ }^{3}$ Serrano Barceló M.M. ${ }^{4}$ \\ ${ }^{1}$ Fisioplanet. Col n990 Illes Balears, Spain \\ ${ }^{2}$ Fisioplanet. Col $n^{\circ} 1341$ Illes Balears, Spain \\ ${ }^{3}$ Fisioplanet. Col n ${ }^{\circ} 1260$ Illes Balears, Spain \\ ${ }^{4}$ Fisioplanet. Col n ${ }^{\circ} 1252$ Illes Balears, Spain \\ Rev Fisioter Invasiva 2019;2:73-74.
}

\begin{abstract}
Introduction and Aims Considering the difficult access of the popliteal muscle (PM) for manual therapy and the proximity of the neurovascular package, we believe it is necessary to perform an exhaustive study in order to describe the dangers of current techniques for approaching this muscle and in order to develop an approach protocol that ensures safety for patients.

Material and Methods We performed a qualitative search in PubMed using the words: popliteus AND muscle, AND/OR treatment AND/OR dry needling, furthermore we consulted two of the most important scientific references on the technique of dry needling. Concurrently, an ultrasound study was performed with the General Electrics Logiq V2 device, with the patient in side lying on the side to be treated. A sample of 4 subjects was examined, obtaining the following measurements: (M1) Distance between the joint interline of the knee and a point at which sonographically the muscle belly of the PM disappears. (M2) Distance between the joint interline of the knee and a point at which the muscle belly of the PM is more accessible to the direct action of a needle. (M3) Ultrasound distance measured from the point at which the muscle belly of the PM is more accessible and which goes from the element of the neurovascular bundle which is closest until the point at which the needle enters the skin. (M4) Ultrasound distance measured from the point at which the PM is more accessible and which includes the space between the neurovascular bundle and the tibia.

Results We were unable to find results in the Pubmed database search related to invasive techniques for the PM. The technique described by the two leading authors on dry needling of the PM is the same; performed without sonographic guidance, guided by muscle palpation (which can lead to less inter-observer agreement) and with needles of up to $50 \mathrm{~mm}$, which could involve a greater risk of needling the vasculonervous bundle according to the measures obtained $(M 3=3.8 \mathrm{~cm})$. The following mean measurements were obtained: (M1) $8.5 \mathrm{~cm}$ (M2) $5.5 \mathrm{~cm}$ (M3)

Keywords

- popliteus

- ultrasonography

- popliteal vein

- popliteal artery

- tibial nerve

$3,8 \mathrm{~cm}(\mathrm{M} 4) 1.6 \mathrm{~cm}$.

Conclusions The current approach on the PM has a real and unnecessary danger of invading vascular and nervous structures and harming the patient. The correct invasive technique for the PM should be performed by placing the patient in side lying on the side to be treated with the knee in semi flexion, sonographically assessing the point where access to the PM muscle belly is best by placing the probe
\end{abstract}

DOI https://doi.org/

$10.1055 / \mathrm{s}-0039-3401857$.

ISSN 2386-4591.
Copyright ( 2019 by Thieme Revinter

Publicações Ltda, Rio de Janeiro, Brazil
License terms

(ㅇ)( $\Theta \Theta$ 
parallel the muscle fibers, performing the entrance along the long axis of the probe, with a maximum needle length of $40 \mathrm{~mm}$. 